Purdue University

Purdue e-Pubs

August 2008

\title{
nanoHUB.org - online simulation and more materials for semiconductors and nanoelectronics in education and research
}

\author{
Gerhard Klimeck \\ Purdue University - Main Campus, gekco@purdue.edu \\ Michael McLennan \\ Purdue University - Main Campus \\ Mark S. Lundstrom \\ Purdue University - Main Campus
}

George B. Adams III

Purdue University - Main Campus

Follow this and additional works at: https://docs.lib.purdue.edu/nanodocs

Klimeck, Gerhard; McLennan, Michael; Lundstrom, Mark S.; and Adams, George B. III, "nanoHUB.org online simulation and more materials for semiconductors and nanoelectronics in education and research" (2008). Other Nanotechnology Publications. Paper 144.

https://docs.lib.purdue.edu/nanodocs/144

This document has been made available through Purdue e-Pubs, a service of the Purdue University Libraries.

Please contact epubs@purdue.edu for additional information. 


\title{
nanoHUB.org - online simulation and more materials for semiconductors and nanoelectronics in education and research
}

\author{
Gerhard Klimeck, Michael McLennan, Mark S. Lundstrom, George B. Adams III. \\ Purdue University, Network for Computational Nanotechnology, West Lafayette, IN 47907 \\ gekco@purdue.edu
}

\begin{abstract}
nanoHUB.org provides a community service to over 65,000 users in over 172 countries annually with "online simulations and and more". Over 85 interactive simulation tools supported by tutorials and general nanotechnology information material are available free of charge to anybody. In all there are over 1,000 resources on nanoHUB. Usage in over 30 classes in the last year and over 270 citations in the literature demonstrate dual usage of nanoHUB in education and research. The content is contributed by members of the Network for Computational Nanotechnology (NCN) which hosts nanoHUB and an increasing number of users. nanoHUB.org is supported by a state-of-the-art content management system and embraces Web 2.0 technologies, that engage the user community through automated contribution processes, tagging, and user ratings. User ratings, usage patterns, and scientific citations of nanoHUB content flow into the content ranking which influences the ranking in nanoHUB searches and category listings. Despite these advanced capabilities, some users still benefit from "personal collections" or "topic pages" by domain experts that aggregate nanoHUB content for specific audiences. Here we highlight the topic pages on "Nanoelectronics", "Non-Equilibrium Green Functions", and "Semiconductor Device Education Materials" as examples relevant to the IEEE nano community.
\end{abstract}

\section{INTRODUCTION}

nanoHUB.org is a freely available resource for research, education and collaboration in nanotechnology developed by the NSF-funded Network for Computational Nanotechnology (NCN). nanoHUB hosts over 1,000 resources which will help users learn about nanotechnology, including Online Presentations, Courses, Learning Modules, Podcasts (which are also cross listed at Apple's iTunes), Animations, Teaching Materials, and more. Most importantly, nanoHUB offers a broad variety of simulation tools, which users can access from their web browser without any software installation on their local machine. All nanoHUB services can be delivered on typical computers with typical Flash and Java enabled browsers. The actual simulations do not run on the user's computer, but on a sophisticated backend that may distribute the computational load to nanoHUB compute resources at Purdue or on the computational grid. This architecture enables the deployment of real research and production codes, not just simple Java applets. nanoHUB also provides collaboration environment via workspaces, online meetings, user groups, and wiki development pages.

From March 2007 to April 2008 over 65,000 users from 172 countries accessed nanoHUB.org [1]. We define a user as a logged-in, self-identified individual, or an IP address that is using interactive seminar content for more than 15 minutes, or an IP address that downloads (not just views) a content item. As of 2007-08, nanoHUB is receiving 3-5 million web hits monthly. Most of our users come from academic institutions and use nanoHUB as part of their research and educational activities. But we also have users from national labs and from industry. The subsequent sections provide a nanoHUB overview, sample application screen shots, and topic pages motivated by the ever increasing nanoHUB content.

\section{NANOHUB TOOLS}

Most of our applications are devoted to nanoelectronics right now, reaching from semiconductor device models to nanowire simulations. The number of applications in the areas of nanoelectromechanics (NEMs), nanodevices for medicine, and nanophotonics are increasing in number. Over 6,200 users have run over 290,000 simulations and over 65,000 users have utilized the nanoHUB in the past 12 months (ending April '08).

The semiconductor simulation tools available on the nanoHUB can address issues in quantum dots, resonant tunneling diodes, carbon nanotubes, $\mathrm{PN}$-junctions, MOS capacitors, MOSFETs, nanowires, ultra-thin-body MOSFETs, finFETs, and others. The nanoHUB simulation facility is different from most other online simulation facilities. The tools are not driven by web-forms with static data output, but by full-fledged, interactive, UNIX-based applications that can be accessed by a web browser. Users can interactively set-up their numerical experiment, view results, compare different simulation runs, and easily ask "What if?" questions. The tools under the hood can be sophisticated industrial device simulation engines, advanced simulation tools, or simple MATLAB scripts that explore concepts. The user is not bothered with the set-up of complicated, arcane input decks, but the tool capabilities are exposed through a graphical user interface. Within the past 3 years, over 85 simulation tools have been deployed.

The Rapid Application Infrastructure [2] (Rappture) has been a key enabler for the development and deployment of these tools. Rappture generates a graphical user interface for any simulation tool automatically, given a description of its inputs and outputs. This toolkit was developed by $\mathrm{NCN}$ and has been released as an open source project on rappture.org [2]. Over 200 projects are managed through our development site nanoFORGE.org [3], and nearly all of these projects use Rappture. 


\section{NANOHUB CONTENT AND MANAGEMENT}

Early on in the NCN project we decided to support the nanotechnology community with more than just the simulation services. The tools need to be supported by tutorials, case studies and scientific documentation. Tutorials on simulation approaches and advanced research seminars were also identified as excellent high value content items. We experimented with various delivery mechanisms including video-based lectures, which demanded a very large communication bandwidth. We decided to focus on the delivery of animated PowerPoint slides that are voiced over through Flash-based technology.

nanoHUB.org is built on the open source Linux+Apache+ MySQL+PHP (LAMP) platform [4] and the Joomla [5] content management system. Use of an open source system allowed us to extend the existing system and customize it to our needs.

By now over 460 contributors have authored over 1,000 nanoHUB content items. With this growth in contributions, we needed to make nanoHUB a self-service publishing venue, with very little administrative overhead. The new contribution mechanisms for the various different nanoHUB content types such as tools, animated lectures, animations, teaching materials, etc. was rolled out into production service in 2007. Simple presentations and documents now just go through a simple administrative quality and completeness check. Even the software application contribution process, which turns out to be the most complicated one, is now automated to a point where little administrative interaction is needed. The software application process is now regulated through several automated stages of project creation, software development, testing, installation, verification, and approval.

The over 1,000 content items need to be manageable by a small deployment team and easily accessible by tens of thousands of users. The nanoHUB team has embraced Web 2.0 technologies such as tagging and rating. Users can tag content items that help the tag-based browsing for all users and the nanoHUB search engine.

Users can also rate content on a 5-star basis and provide textual feedback. User ratings, content usage, and content citation in the research literature flow into a formula that ranks nanoHUB content on a scale from 0 to 10 . The ranking influences nanoHUB search engine results presentation order.

\section{NANOHUB TOPIC PAGES}

Even with the state-of-the-art Web 2.0 technologies we have found that users may need easy-to-use summary pages that are procured by a "trusted" authority. While we are still experimenting with the technology that enables the author or the small set of authors to edit these topic pages, we have deployed three pages and are beginning to monitor their usage.

With the proliferation of nanoelectronic-related content on nanoHUB, users have requested help in identifying the content best suited for specific purposes. Prof. Mark Lundstrom, NCN Director, has developed the Nanoelectronics Topic Page [6] to provide an overview of the applications and developments of the NCN Nanoelectronics thrust. This page provides Selected Resources such as Tutorials, Research Seminars, Courses, and Simulation Tools and Special Initiatives such as Concepts of Quantum Transport, Electronics from the Bottom Up, and the NEGF Resource Page [7].

In the past 10-15 years the non-equilibrium Green function formalism (NEGF) has begun to distinguish itself as the fundamentally sound formalism to treat non-equilibrium carrier transport on the nanometer scale fully quantum mechanically. While the formalism was developed in the Physics community in the 1950s, the resulting literature has been extremely difficult to penetrate for nanoelectronic device engineers and physicists. Prof. Supriyo Datta at Purdue University is recognized as a leader in the theory of electron transport and he is now assembling references to nanoHUB content he deems essential to the introduction to and understanding of NEGF. The NEGF page [7] coexists with the automatically generated nanoHUB page that is based on the tag "negf".

From informal collegial feedback we have found that a common usage scenario is that of a faculty member wanting to supplement an existing course with interactive simulations. The required materials are neither full courses, nor full lectures, but lecture elements, homework problems, or project assignments. The access to such course elements must be rapid and not involve searching and aggregation. With the strength in semiconductor devices and nanoelectronics we have identified a type of course for which we provide a prototype of such course material aggregation; almost every electrical engineering program around the world teaches an introductory semiconductor device course at the undergraduate and/or graduate level. This is the target of the "Semiconductor Device Education Materials" topic page [8] that is maintained by Prof. Gerhard Klimeck at Purdue.

\section{SEMiconductor DEVICE EdUCATION MATERIALS}

Almost every Electrical Engineering department teaches the fundamental concepts of semiconductor devices. These

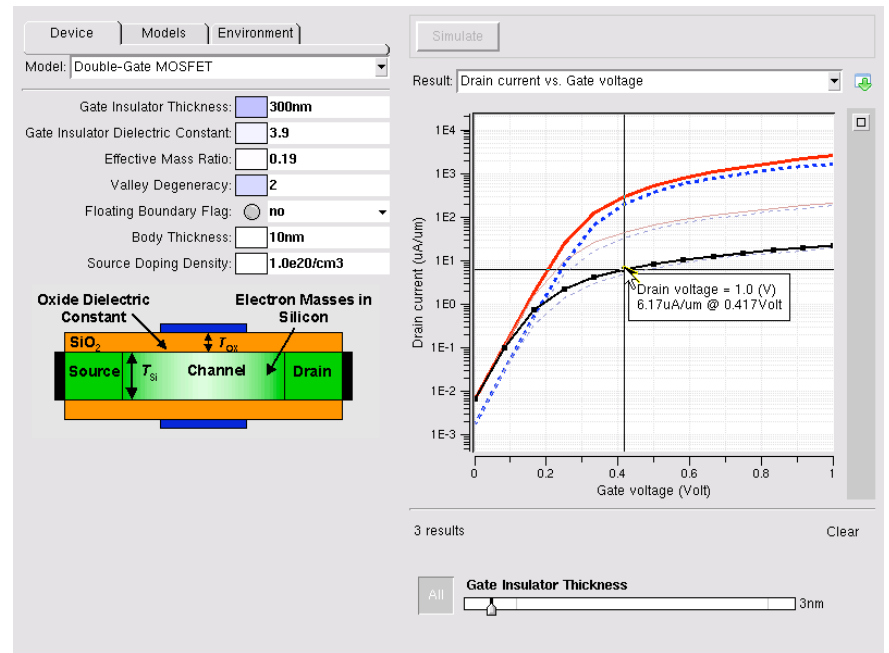

Fig. 1. Screen shot of the FETtoy tool. Users can modify input parameters and compare various simulation results with the data sliders. 
concepts typically include lattices, crystal structure, bandstructure, band models, carrier distributions, drift, diffusion, P-N junctions, solar cells,light-emitting diodes, bipolar junction transistors (BJT), metal-oxide semiconductor capacitors (MOS-cap), and multi-acronym-device field effect transistors (mad-FETs). Advanced courses go more deeply into semiconductor theory, device physics, fabrication processes, and advanced and special purpose devices, such as heterostructures, power devices, and optoelectronic devices. This nanoHUB "topic page" provides an easy access to selected nanoHUB Semiconductor Device Education Material.

The next paragraphs highlight the many nanoHUB semiconductor applications. Many tools are supplemented by tutorials, homework assignments, and project suggestions. The user community is invited to provide further associated materials through the open nanoHUB contribution process.

\section{A. Crystal Structures, Lattice}

The Crystal Viewer tool enables the interactive visualization different Bravais lattices, and crystal planes, and materials (diamond, Si, InAs, GaAs, graphene, buckyball).

\section{B. Band Models / Band Structure}

The Periodic Potential Lab solves the time independent Schrödinger Equation in a 1-D spatial potential variation. Rectangular, triangular, parabolic (harmonic), and Coulomb potential confinements can be considered. The user can determine energetic and spatial details of the potential profiles, compute the allowed and forbidden bands, plot the bands in a compact and an expanded zone, and compare the results against a simple effective mass parabolic band. Transmission is also calculated through the well for the given energy range.

Bandstructure Lab supports the study of bulk dispersion relationships of $\mathrm{Si}$, GaAs, InAs. Users can apply tensile and compressive strain and observe the variation in the bandstructure, bandgaps, and effective masses. Advanced users can study bandstructure effects in ultra-scaled (thin body) quantum wells, and nanowires of different cross sections. Bandstructure Lab uses the $\mathrm{sp} 3 \mathrm{~s} * \mathrm{~d} 5$ tight binding method to compute $\mathrm{E}(\mathrm{k})$ for bulk, planar, and nanowire semiconductors.

StrainBands uses first-principles density functional theory within the local density approximation and ultrasoft pseudopotentals to compute and visualize density of states, $\mathrm{E}(\mathrm{k})$, charge densities, and Wannier functions for bulk semiconductors. Using this tool, users can study and learn about the bandstructures of bulk semiconductors for various materials under hydrostatic pressure and under strain conditions. Physical parameters such as the bandgap and effective mass can also be obtained from the computed $\mathrm{E}(\mathrm{k})$. We note here that the bandgaps obtained with DFT-LDA are underestimated, by about a factor of two for some semiconductors (including $\mathrm{Si}$ and GaAs), as is well known.

\section{Carrier Distributions}

Carrier Statistics Lab demonstrates electron and hole density distributions based on the Fermi-Dirac and Maxwell Boltzmann equations. It shows the dependence of carrier density, density of states and occupation factor on temperature and Fermi level. Users can choose between doped and undoped semi-conductors. Silicon, Germanium, and GaAs can be studied as a function of doping or Fermi level, and temperature.

\section{Bulk Semiconductors - Drift Diffusion}

Drift Diffusion Lab helps its users understand the concepts of drift and diffusion of carriers inside a semiconductor slab. Experiments like shining light on the semiconductor, applying bias and both can be performed. This tool provides important information about carrier densities, transient and steady state currents, Fermi levels and electrostatic potentials.

\section{E. Semiconductor Process Modeling}

Semiconductor process modeling is a vast field for which several commercial products are available and in production use in industry and, to some extent, in education. nanoHUB is serving a few applications that are primarily geared towards education. The four tools entitled Process Lab (PL) 'PL Oxidation', 'PL Oxidation Flux', 'PL Concentration Dependent Diffusion', and 'PL Point Defect Coupled Diffusion' are all educational front-ends to the general PROPHET tool.

\section{F. PN Junctions}

$P N$ Junction Lab provides everything users need to explore the basic concepts of P-N junction devices. Users can edit the doping concentrations, change the materials, tweak minority carrier lifetimes, and modify the ambient temperature. Then, they can see the effects in the energy band diagram, carrier densities, net charge distribution, I/V characteristic, etc. There is an extensive set of associated resources available for this tool, such as demos, learning module, and homework assignments.

\section{G. Bipolar Junction Transistors}

The Bipolar Junction Transistor Lab uses a 2D mesh and allows users to simulate npn or pnp type devices. Users can specify the Emitter, Base and Collector region depths and doping densities. Also material and minority carrier lifetimes can be specified. It is supported by a homework assignment in which students are asked to find the emitter efficiency, the base transport factor, current gains, and the Early voltage.

\section{H. MOS Capacitors}

The MOScap tool enables a semi-classical analysis of MOS Capacitors. It simulates the capacitance of bulk and dual gate capacitors for a variety of different device sizes, geometries, temperature and doping profiles.

SCHRED calculates the envelope wavefunctions and the corresponding bound-state energies in a typical MOS (MetalOxide-Semiconductor) or SOS (Semiconductor-OxideSemiconductor) structure and a typical SOI structure by solving self-consistently the one-dimensional (1D) Poisson equation and the $1 \mathrm{D}$ Schrodinger equation.

\section{I. mad-FETs (multi-acronym device FETS)}

The MOSFET tool computes a semi-classical analysis of current-voltage characteristics for bulk and SOI Field Effect Transistors (FETs) for a variety of different device sizes, geometries, temperature and doping profiles. 
nanoMOS provides a 2D simulation for thin body MOSFETs, with transport models ranging from drift-diffusion to quantum diffusive for a variety of different device sizes, geometries, temperature and doping profiles.

nanoFET Lab simulates quantum ballistic transport properties in two-dimensional MOSFET devices for a variety of different device sizes, geometries, temperature and doping profiles.

FETToy 2.0 is a set of MATLAB scripts that calculate the ballistic I-V characteristics for a conventional MOSFETs, Nanowire MOSFETs and Carbon NanoTube MOSFETs. For conventional MOSFETs, FETToy assumes either a single or double gate geometry and for a nanowire and nanotube MOSFETs it assumes a cylindrical geometry. Only the lowest subband is considered, but it is readily modifiable to include multiple subbands.

\section{J. Technology Computer Aided Design (TCAD) Simulators}

$P A D R E$ is a $2 \mathrm{D} / 3 \mathrm{D}$ simulator for electronic devices, such as MOSFET transistors. It can simulate physical structures of arbitrary geometry, including heterostructures, with arbitrary doping profiles, which can be obtained using analytical functions or directly from multidimensional process simulators such as PROPHET.

PROPHET was originally developed for semiconductor process simulation. PROPHET solves sets of partial differential equations in one, two, or three spatial dimensions. The equations to be solved can be specified by the end user. It is supported by an extensive set of User Guide pages and a seminar on Nano-Scale Device Simulations Using PROPHET.

\section{CONCLUSIONS}

nanoHUB.org is a community web site serving a growing number of users worldwide (over 65,000 annual users as of April 2008) with a growing number of content items (over 1,000). We can document the usage of each tool and have found for the tools that we converted from web-forms to fully interactive tools with the ability to ask "What if?" questions, usage increased dramatically. Table I shows the user and usage statistics of the simulation tools that are featured in the Semiconductor Device Education Materials Page. The comparison between the true total user count (last row) and the simple sum of the individual tool users shows that users on average use 2 tools. We have found that no distinction can be made a priori between educational use or research use of the simulation tools. For example the SCHRED, nanoMOS, and FETtoy tools are clearly a dual use tool for education with documented classroom use and research as documented with 74,41 , and 11 citations in the research literature, respectively (see Table 1). The key element to the nanoHUB growth is true usability. Here we describe a new element of the nanoHUB that increases its usability: topic pages geared for particular audiences.
TABLE I

TOOL USER AND USAGE STATISTICS FOR TOOLS REFERENCED IN THE MATERIALS FOR SEMICONDUCTOR EDUCATION

\begin{tabular}{|l|r|r|r|r|r|r|}
\hline Toul Name & $\begin{array}{c}\text { Service } \\
\text { Months }\end{array}$ & $\begin{array}{r}\text { Annual } \\
\text { Users }\end{array}$ & $\begin{array}{c}\text { Annual } \\
\text { Sim. }\end{array}$ & $\begin{array}{c}\text { Total } \\
\text { Users }\end{array}$ & $\begin{array}{c}\text { Total } \\
\text { Sims }\end{array}$ & $\begin{array}{c}\text { Total } \\
\text { Citations }\end{array}$ \\
\hline Adept & 12 & 274 & 5,975 & 589 & 17,056 & 1 \\
\hline Bandstructure & 24 & 864 & 6,360 & 1,656 & 11,093 & 3 \\
\hline BJT & 2 & 65 & 298 & 65 & 298 & \\
\hline Carrier Stat. & 4 & 90 & 560 & 90 & 560 & \\
\hline Crystal Viewer & 4 & 204 & 1,016 & 204 & 1,016 & \\
\hline Drift-Diffusion & 3 & 204 & 1,016 & 204 & 1,016 & \\
\hline FETtoy & 27 & 477 & 5,792 & 1,489 & 24,931 & 11 \\
\hline MOScap & 25 & 356 & 3,048 & 691 & 6,419 & \\
\hline MOSFET & 26 & 812 & 9,608 & 1,534 & 17,425 & \\
\hline nanoFET & 15 & 313 & 1,025 & 421 & 10,548 & 1 \\
\hline nanoMOS & 24 & 364 & 2,588 & 1,025 & 8,843 & 41 \\
\hline Padrc & 28 & 234 & 10,848 & 497 & 15,337 & \\
\hline Periodic Pot. & 3 & 90 & 560 & 90 & 560 & \\
\hline PL - Conc.D.D & 19 & 177 & 5,925 & 364 & 12,904 & \\
\hline PL - Oxidation & 19 & 191 & 5,911 & 417 & 15,387 & \\
\hline PL - Ox. Flux & 19 & 101 & 808 & 232 & 2,196 & \\
\hline PL- Pnt Def.D & 19 & 55 & 336 & 137 & 1,150 & \\
\hline PN Junction & 32 & 624 & 5,195 & 1,905 & 16,734 & \\
\hline Prophet & 36 & 109 & 1,520 & 479 & 6,656 & 2 \\
\hline SCHRED & 27 & 527 & 7,673 & 1,246 & 30,046 & 74 \\
\hline Strain Bands & 11 & 204 & 1,016 & 204 & 1,016 & \\
\hline Simple Sum & & 6,335 & 77,078 & 13,539 & 201,191 & 133 \\
\hline True Users & & 3,219 & & 6,905 & & \\
\hline
\end{tabular}

\section{ACKNOWLEDGMENT}

We gratefully acknowledge the work of the nanoHUB software team, the over 460 content contributors to date, and the leadership of the NSF program managers and directors who saw the potential of this work. NCN is primarily funded by the National Science Foundation under Cooperative Agreement EEC-0634750 and grant OCI-0721680.

The text in Section V on Semiconductor Device Education is derived from the associated page [8] on nanoHUB.org, is licensed under creative commons [9], and IEEE does not hold the copyright.

\section{REFERENCES}

[1] http://nanohub.org/usage.

[2] M. McLennan, "The Rappture Toolkit," http://rappture.org (2004).

[3] nanoHUB project development site $\mathrm{http} / / /$ nanoFORGE.org is also aliased as http://developer.nanohub.org.

[4] G. Lawton, "LAMP lights enterprise development efforts," IEEE Computer 38(9), 18-20, (2005).

[5] Available online at http://www.joomla.org/.

[6] Nanoelectronics page http://nanohub.org/topics/ncn nanoelectronics.

[7] NEGF page at http://nanohub.org/topics/negf.

[8] Available online at http://nanohub.org/topics/edu semiconductor.

[9] Available online at http://creativecommons.org/licenses/by-nc-sa/2.5/ 